\title{
Idiopathic dilatation of the right atrium: A case report
}

\author{
Valentin Sinitsyn ${ }^{1}$, Elena Mershina ${ }^{1}$, Ekaterina Bazaeva ${ }^{2}$ and Roman Myasnikov ${ }^{2}$ \\ ${ }^{1}$ Federal Center of Treatment and Rehabilitation, Ivan'kovsloye sh.3, Moscow, 125367, Russia \\ ${ }^{2}$ National Research Center for Preventive Medicine, Bld. 10, Petroverigskiy lane, Moscow, 101990, Russia
}

\begin{abstract}
We report a rare case of idiopathic prominent dilatation of the right atrium (RA) in an adult female. Detailed cardiological and radiological (including cardiac CTA and MRI) examinations demonstrated absence of atrial septal defect, Ebstein's anomaly, tricuspid or pulmonary valve stenosis or any other diseases leading to RA enlargement. The RA has diameter up to $10 \mathrm{~cm}$ and volume around $400 \mathrm{ml}$. Its size was out of proportion to size of both ventricles and left atrium. The patient had a familial history of cardiac deaths because of some unknown cardiac diseases manifested as heart failure. There are rare case reports of idiopathic dilatation of the RA in the literature. This rare pathology should be considered in cases of severe disproportional enlargement of RA.
\end{abstract}

\section{Introduction}

In clinical settings enlargement of the left atrium (LA) occurs more frequently than of the right atrium (RA). In most cases enlargement of the RA is related to congenital or acquired disease of the tricuspid valve (Ebstein anomaly, stenosis or insufficiency of the valve) or volume overload due to atrial septal defect with left-to-right shunting of the blood. But some rare cases of idiopathic dilatation of the RA (both sporadic and familial) have been described in the literature [1,2]. We present a case of a giant RA in an adult patient without signs of any other disease which could be related to this abnormality.

\section{Case report}

A 63-year old female was referred to cardiac MRI because of increasing symptoms of right heart failure and dilatation of the RA found on echocardiography. Her complains were general fatigue, dyspnea on exercise, swelling of lower extremities and enlargement of the abdomen. She was asymptomatic till the year of 2007, when permanent form of atrial fibrillation appeared and she experienced first signs of general fatigue which increased over time. During one of her hospitalization a diagnosis of acute myocardial infarction was suspected because of abnormal ECG, but it had not been verified with coronary angiography or troponin test. Her mother died at the age 53 because of some unknown cardiac disease, her son suffers from atrial fibrillation, on echocardiography he has dilatation of both atria without enlargement of ventricles. Since 2011 she complains of increased volume of abdomen and legs swelling. A diagnosis of chronic myocardial infarction and liver cirrhosis was established by her attending physician. On physical examination her BP was $110 / 70 \mathrm{Hg}$, heart rate $72 \mathrm{bpm}$, irregular, there was a systolic murmur on auscultation. Her BMI was 25 , there were symptoms of liver enlargement, ascites, edema of lower extremities, and her neck veins were distended.

ECG recorded atrial fibrillation with heart rate $70-85 \mathrm{bpm}$, vertical heart axis, ST was elevated up to $1 \mathrm{~mm}$ in V4-5 and depressed in II, III, aVF. 24-hour Holter monitoring of ECG recorded atrial fibrillation with average rate $83 \mathrm{bpm}$ (min-max $47-89 \mathrm{bmp}$ ), 566 polytopic polymorphic ventricular extrasystole.

Blood test were in normal ranges except for mild signs of decreased liver function (low total cholesterol and protein, increased level of alkaline phosphatase). Count of eosinophils in the peripheral blood was normal. No serological markers of carcinoid syndrome were found.

Transthoracic echocardiography (TTE) demonstrated severely increased RA (long and short axis, were 79 and $95 \mathrm{~mm}$, correspondingly). Marked tricuspid regurgitation with dilatation of valve annulus was recorded. Right ventricle (RV) was moderately enlarged (end-diastolic diameter (EDD) was $5.8 \mathrm{~cm}$ ) with normal values of ejection faction (EF) - 45-46\%. Left atrium (LA) was mildly enlarged $(5.5 \times 7.6 \mathrm{~cm})$, there was mild mitral regurgitation. Diameter of pulmonary artery was normal (3 $\mathrm{cm})$. Left ventricle (LV) was not enlarged (EDD was $5 \mathrm{~cm}$, end-systolic diameter $4 \mathrm{~cm}$ ), but ejection fraction (EF) was moderately decreased to $45 \%$ due to global hypokinesia of LV myocardium. Thicknesses of intraventricular septum (IVS) and LV posterior wall (LVPW) were 10-12 $\mathrm{mm}$. No anatomical abnormalities of atrio-ventricular, aortic or pulmonary valves were found. No signs of pericardial effusion.

In order to clarify results of echocardiography, a cardiac MRI with late gadolinium enhancement (LGE) was performed with $1.5 \mathrm{~T}$ scanner (Avanto, Siemens AG). Results of MRI were similar to ones of echocardiography. Black blood MR and cine-MR revealed that RA was prominently enlarged (diameter up to $10 \mathrm{~cm}$, volume $400 \mathrm{ml}$ ) (Figures 1 and 2).

Correspondence to: Dr. Valentin Sinitsyn, Federal Center of Treatment and Rehabilitation, Ivan'kovsloye sh.3, Moscow, 125367, Russia, Tel: +7 495 9424020; E-mail: vsini@mail.ru

Key words: heart, right atrium, idiopathic, dilatation, cardiomyopathy, fibrillation, MRI, CTA

Special Issue: Imaging of myocardial diseases: to new horizons

Valentin E. Sinitsyn

Professor

Department of Radiology

Federal Center of Medicine and Rehabilitation

Russia

E-mail: vsini@mail.ru

Published: July 30, 2016 


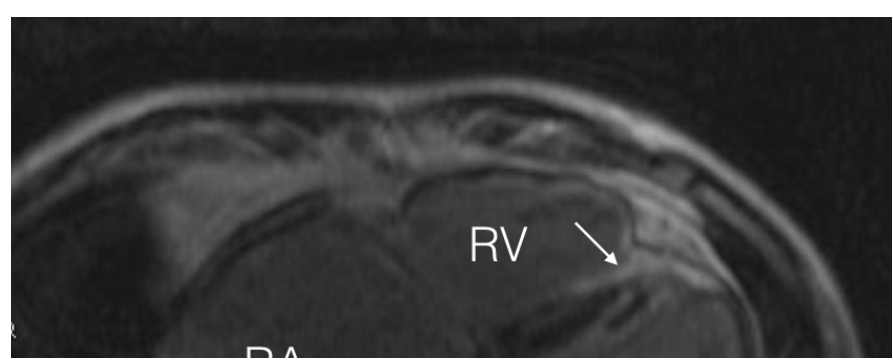

Figure 1. Black-blood TSE MRI. Left side - axial slice, right side - coronal slice. Prominen enlargement of RA is seen.

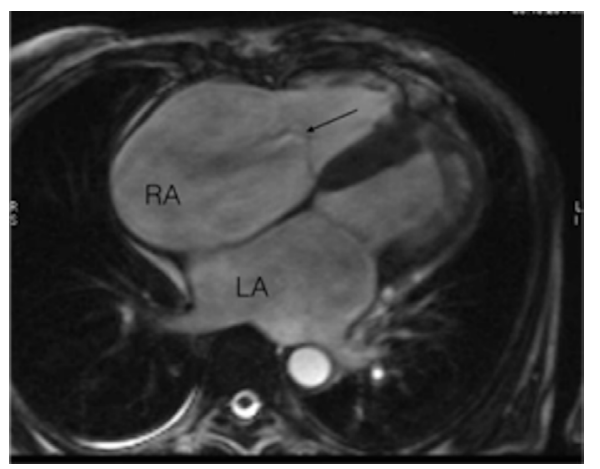

Figure 2. Cine-MR, four-chamber view. Marked dilatation of RA, moderate enlargemen of LV. Arrow shows a jet of tricuspid regurgitation and normal position of valve leaflets.

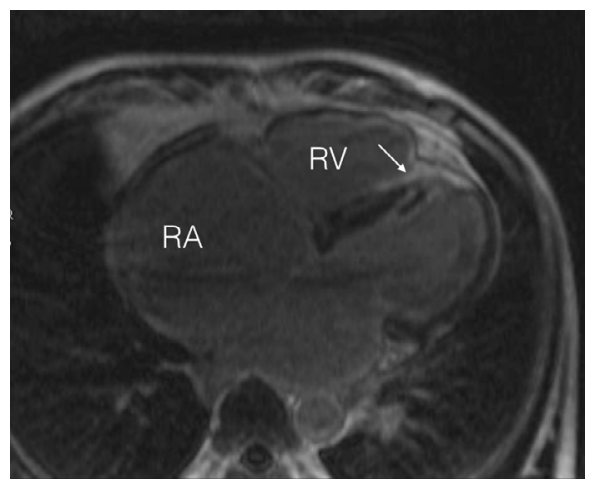

Figure 3. LGE MRI, four-chamber view. Arrow indicates focal area of RV endocardial surface of IVS. Two short arrows mark a thrombus inside LV cavity.

There were mild dilatation of RV (end-diastolic volume (EDV) 115 $\mathrm{ml} / \mathrm{m} 2$ (upper range of normal value $100 \mathrm{ml} / \mathrm{m}^{2}$ ) and LV (EDV $95 \mathrm{ml} /$ $\mathrm{m}^{2}$, upper range of normal value $\left.92 \mathrm{ml} / \mathrm{m}^{2}\right)$. LA was mildly enlarged $(5,5$ $\mathrm{cm}$ in diameter). MRI demonstrated mild mitral and severe tricuspid regurgitation. Thickness of RV myocardium was in normal range (3-4 $\mathrm{mm})$. IVS was thicker (13-14 $\mathrm{mm})$ than LVPW $(10 \mathrm{~mm})$. LV EF was decreased to $44 \%$.

LGE MR demonstrated a focal area of contrast enhancement (inside the right side of IVS - Figure 3) representing the subendocardial fibrosis and an ovoid structure inside LV cavity fixed between the papillary muscle and IVS. No signs of any congenital heart disease (first of all, Ebstein's anomaly, atrial septal defect, anomalous venous drainage) were found. Anatomy of valves was normal.

In order to clarify the status of coronaries and an intracavitary structure inside the LV, coronary CTA (followed by venous-phase cardiac CT) was performed after MRI. Low-dose CTA was done with 64-row scanner (Discovery HD750, GE Healthcare) using prospective gating and tube voltage $80 \mathrm{kV}$. Coronary CTA showed mild nonsignificant calcified coronary plaques in left anterior descending artery (LAD) and a calcified ovoid structure (chronic thrombus) inside the LV cavity. Leaflets of all valves were of normal thickness. No signs of acute or chronic pulmonary thromboembolism were found. Lung fields were without remarkable pathology.

Abdominal MRI and CT revealed accepts and diminished size of the right lobe of the liver, which also had uneven contours, dilated inferior vena cava and hepatic veins. No tumors in the liver or other abdominal organs were found Mediastinal and abdominal lymph nodes were not enlarged. The spleen was of normal size, diameters of portal and splenic veins were in normal ranges. The changes of the liver and veins were interpreted as manifestations of cardiac cirrhosis (congestive hepatopathy) due to long-term right-heart failure (Figures 4 and 5).

\section{Discussion}

Usually enlargement of the RA is secondary to tricuspid valve disease, pressure or volume overload of the right heart chambers due to congenital or acquired diseases. But in this patient secondary nature of severe RA enlargement was excluded. Such configuration of the heart is not typical for arrhythmogenic right ventricular cardiomyopathy (ARVC). Formally she had one major criteria of ARVC (mild enlargement of RV). But no other criteria of ARVC were found (first of all, the patient had not arrhythmia characteristic for this disease). Disproportional (in comparison to RV, LA and LV) dilatation of RA may be observed in some forms of primary or secondary restrictive cardiomyopathies. Pericardial diseases were excluded. Due to very large RA and signs of focal endocardial fibrosis detected with LGE MRI, a possibility of secondary restrictive cardiomyopathy due to carcinoid syndrome was considered. But scrupulous search for any neuroendocrine tumors or metastatic lesions brought no results.
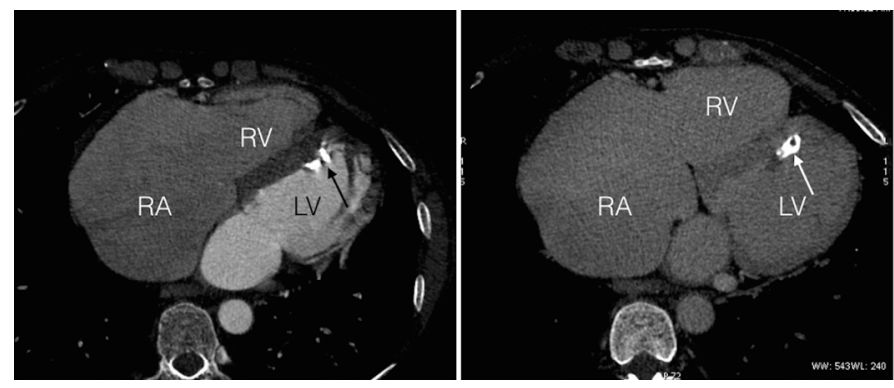

Figure 4. Cardiac CT. Left side - arterial phase (CTA), right side - venous phase. Marked enlargement of RA, leaflets of tricuspid valve are not thickened or calcified. A calcified thrombus (arrow) is seen inside LV attached to IVS.

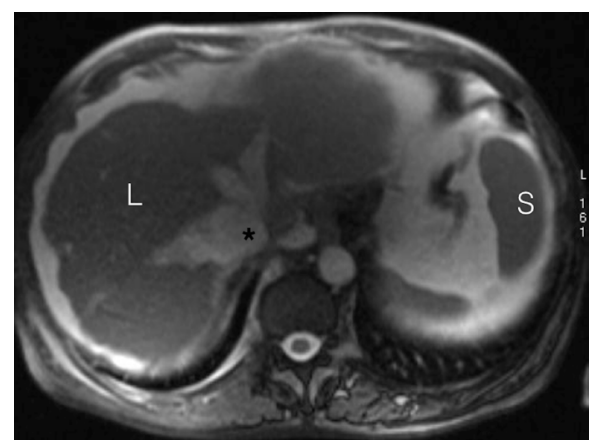

Figure 5. Abdominal MRI, T2-weighted image. L - right lobe of the liver, surrounded with ascites, $\mathrm{S}$ - spleen, asterisk - dilated inferior vena cava. 
Besides that, no signs of tricuspid valve fibrosis were found. Most probably that severe tricuspid regurgitation was caused by prominent enlargement of RA and tricuspid. The origin of calcified thrombus inside the almost unaffected LV remains unknown.

So in this case the most probable diagnosis was idiopathic enlargement of the RA. This is a very rare cardiac anomaly - there are less than 100 cases of this disease described in the world literature [3-6]. For example, in year 2000 in review of Binder et al. 60 cases of idiopathic RA enlargement were reported [7]. The diagnosis of this disease is made by comprehensive exclusion of more frequent causes of RA enlargement. In some cases a family cluster of the disease could be followed-up [3] (it is highly probable that the disease was of genetic etiology in this case too). There is a probability that such cases of prominent idiopathic dilatation of RA represent some form of socalled atrial cardiomyopathy. Ventricular (mostly) cardiomyopathies are well studied but very little is known about atrial ones. Some opinion leaders believe that they exist as a separate nosological unit and atrial fibrillation is their major clinical manifestation. This year the first consensus paper on atrial cardiomyopathies has been published [8]. It gives definition of atrial cardiomyopathy as 'any complex of structural, architectural, contractile or electrophysiological changes affecting the atria with the potential to produce clinically-relevant manifestations'. The paper is mostly concentrated on LA atrial fibrosis and atrial fibrillation. But may be in the following publications cases of idiopathic RA enlargement could be included into this diagnostic entity. Due to rarity of this disease, there are very little evidence-based data about management and treatment of it. Symptomatic therapeutic approach is targeted to compensation of the right heart failure. Probably the best approach in symptomatic patients is cardiac surgery directed to reduction of RA size and correction of secondary tricuspid regurgitation (annuloplasty, valve replacement).

\section{Conclusion}

Idiopathic dilatation of RA is a very rare disease. Nevertheless probability of this pathology should be kept in mind when usual etiologies of RA enlargement are excluded. Cardiac imaging (first of all, MRI and echocardiography) plays a pivotal role in diagnosis. There is probability that such cases represent a specific form of atrial cardiomyopathy. Further collection and analysis of similar cases will help to understand better this unusual pathology of RA.

\section{Acknowledgements}

We thank the staff of the Department of Radiology of Federal Center of Treatment and Rehabilitation for help and assistance.

\section{References}

1. Blondheim DS, Klein R, Marmor AT (2000) Familial idiopathic dilation of the right atrium with complete atrioventricular block: a new syndrome? Cardiology 94: 224 226.[Crossref]

2. Chatrath R, Turek O, Quivers ES, Driscoll DJ, Edwards WD, et al. (2001) Asymptomatic giant right atrial aneurysm. Tex Heart Inst J 28: 301-303.[Crossref]

3. Biocic S, Đurasevic Z, Vincelj J, Udovicic M (2010) A familial cluster of idiopathic dilatation of the right atrium-A two-case report. Journal of Cardiology Cases 2: e52 - e54.

4. Kurz DJ, Oechslin EN, Kobza R, Jenni R (2004) Idiopathic enlargement of the right atrium: 23-year follow up of a familial cluster and their unaffected relatives. Heart 90: 1310-1314. [Crossref]

5. Gomes S, Wolfenden H, Lambros J (2012) Giant right atrium in an adult: case report of a rare condition. Heart Lung Circ 21: 50-52.[Crossref]

6. Long MA (2014) Giant right atrium: an extreme case of idiopathic dilation of the right atrium. Ann Thorac Surg 98: 1815-1818.[Crossref]

7. Binder TM, Rosenhek R, Frank H, Gwechenberger M, Maurer G, et al. (2000) Congenital malformations of the right atrium and the coronary sinus: an analysis based on 103 cases reported in the literature and two additional cases. Chest 117: 1740-1748. [Crossref]

8. Goette A, Kalman JM, Aguinaga L, Akar J, Cabrera JA, et al. (2016) EHRA/HRS APHRS/SOLAECE expert consensus on Atrial cardiomyopathies: definition, characterization, and clinical implication. Europace. [Epub ahead of print]

Copyright: (C2016 Sinitsyn V. This is an open-access article distributed under the terms of the Creative Commons Attribution License, which permits unrestricted use, distribution, and reproduction in any medium, provided the original author and source are credited. 